\title{
Antigen-antibody complexes in the serum of patients with juvenile chronic arthritis
}

\author{
H. MORAN, B. M. ANSELL, J. F. MOWBRAY, R. J. LEVINSKY, AND J. F. SOOTHILL \\ The Hospital for Sick Children, Institute of Child Health, and St Mary's Hospital Medical School, London
}

SUMMARY IgG-containing antigen-antibody complexes were detected in the sera of all of 7 patients with the systemic form of juvenile chronic arthritis, usually at high levels. Only 9 of 25 patients with the polyarticular form had such complexes, usually at low levels. Three patients had low levels of serum IgA which were probably drug induced; the one patient with low $\mathrm{C} 2$ and the 3 with low yeast opsonisation were probably not more than to be expected in a random population.

The aetiology and pathogenesis of juvenile chronic arthritis (JCA) remains unknown, but it has a number of features in common with chronic antigenantibody complex disease-especially the joint, eye, and skin manifestations. Deposition of immunoglobulin and complement in the joints (Munthe and Natvig, 1972) is consistent with this, but we know of only one report of tests for presumed antigenantibody complexes (aac) in the blood of patients with JCA (Rossen et al., 1977) and they were confined to those with the systemic form of the disease. We therefore report our results of IgG containing aac in patients with systemic and polyarticular JCA.

It has been suggested that persistent circulation of aac results from defects of the clearance functions -antibody, complement, and phagocytes (Soothill and Steward, 1971), and immunodeficiency, especially of $\operatorname{IgA}$, is associated with a range of immunopathological diseases (Amman and Hong, 1971; Soothill, 1976). IgA deficiency is found in a significant minority of JCA patients, apparently as a primary defect (Cassidy et al., 1977; Barkley et al., 1978). Low levels of the second component of complement (C2), interpreted as indicating heterozygosity for the deficiency, have also been reported in association with JCA (Glass et al., 1976). Other complement components are usually normal although

\footnotetext{
The Hospital for Sick Children, Great Ormond Street, London H. MORAN, honorary clinical assistant

B. M. ANSELL, honorary consultant physician

Institute of Child Health, London

J. F. SOOTHILL, professor of immunology

R. J. LEVINSKY, research fellow

St Mary's Hospital Medical School, London

J. F. MOWBRAY, professor of imnunopathology
}

some variation occurs during active disease (Bianco et al., 1971). A common defect, apparently of the alternative pathway of complement, detected by defective opsonisation for yeast phagocytosis, is associated with a similar range of diseases as is IgA deficiency (Miller et al., 1968; Soothill and Harvey, 1976, 1977). We have therefore measured, in addition to circulating aac, total haemolytic complement, $\mathrm{C} 2$, $\mathrm{C} 3$, and $\mathrm{C} 4, \operatorname{IgA}, \operatorname{IgG}$, and IgM, and the opsonisation of yeast for phagocytosis by normal polymorphonuclear leucocytes, in the JCA patients.

\section{Materials}

42 patients suffering from JCA (for diagnostic and classification criteria see Ansell and Wood, 1976), age range 6 to 15 years, were studied. 30 had polyarticular disease-23 seronegative and 7 seropositive; 7 had acute systemic disease (they were younger than the rest-age range 4 to 11 years), and 5 had amyloidosis complicating seronegative systemic disease. All were established patients receiving various forms of treatment. Sera from 19 children (age range 2 to 13 years) in hospital for nonimmunological disease (largely cold surgery) were also studied.

\section{Methods}

Antigen-antibody complexes were measured by the method of Levinsky and Soothill (1977). EDTA was added to serum separated within 3 hours of blood collection, and the released $\mathrm{Clq}$ was removed by reaction with insolublised IgG. The serum was then added to a low affinity rabbit IgM antihuman IgG reagent, and the residual capacity of the latter to 
agglutinate $\operatorname{IgG}$ coated latex, was measured by counting unagglutinated particles on a Coulter counter. Total complement was measured using an autoanalyser haemolytic method (modified from Nydegger et al., 1972). It was also adapted for measuring $\mathrm{C} 2$, using mixtures of a C2-deficient serum (ED), and the test sample. Results were expressed as \% of standard. $\operatorname{lgG}, \operatorname{IgA}, \operatorname{IgM}$, and $\mathrm{C} 3$ were measured by a modification of the radial diffusion method of Fahey and McKelvey (1965). Yeast opsonisation was measured by the method of Soothill and Harvey (1976). Results were expressed as opsonisation index (yeasts per polymorph).

\section{Results}

The Figure shows the levels of aac in the sera of patients and controls. The maximum inhibition of agglutination by the latter was $20 \%$, which is consistent with the published upper limit of normal (Levinsky and Soothill, 1977). 10 of the 23 patients with seronegative polyarticular disease had raised levels; in only 3 was the value greater than $30 \%$. All 7 patients with systemic disease had raised levels of aac which were considerably higher than those with polyarticular disease. None of the patients with seropositive disease or amyloidosis had raised levels.

Immunoglobulin and complement were measured in 30 patients with polyarticular disease, positive or negative for rheumatoid factor. $\mathrm{IgG}, \mathrm{IgM}, \mathrm{C3}$, and haemolytic complement values were either normal or high compared with the age-related normal ranges

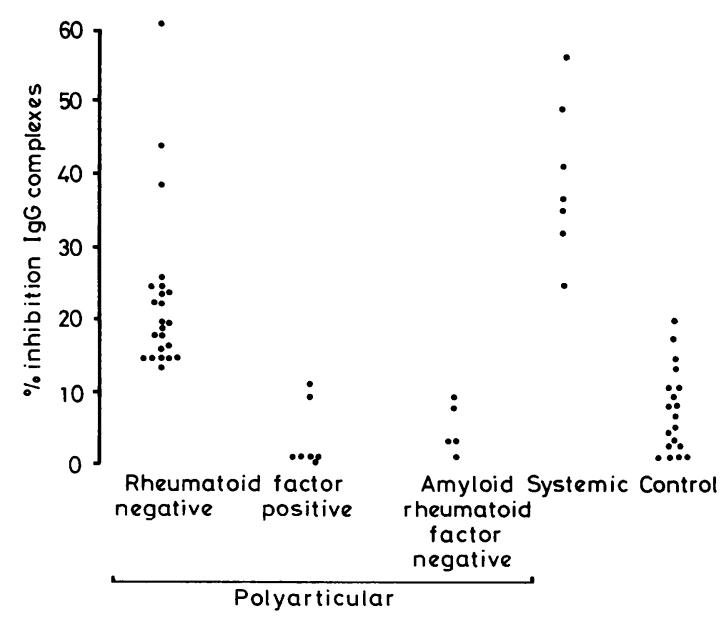

Figure. Levels of IgG-containing antigen-antibody complexes in children with polyarticular and systemic juvenile chronic polyarthritis expressed as percentage inhibition of agglutination of IgG coated latex by rabbit IgM antihuman $\lg G$ reagent. used in the department. This is consistent with previous reports in JCA. IgA was low in 3 patients, all of whom were receiving drugs which could have explained it, and yeast opsonisation was low $(<2 \cdot 5 \mathrm{Y} / \mathrm{P})$ in $3(2 \cdot 30,2 \cdot 10$, and $1 \cdot 60)$; all these 6 had polyarticular seronegative disease.

\section{Discussion}

The high levels of IgG-containing aac in the serum of patients with the systemic form of JCA is consistent with the previous report (Rossen et al., 1977), and with the hypothesis that they may play a part in the pathogenesis of this disease. In contrast, fewer than half of the patients with the seronegative polyarticular form of JCA had raised levels of aac and they had relatively low levels. This casts doubt on their pathogenetic role for this disease but, as the patients were studied at different stages of the disease, and on different treatments, these data do not refute that hypothesis. Antiglobulin antibodies (rheumatoid factor, and others) are low affinity antibodies to human IgG. Since these will agglutinate latex particles, they could produce false-negative results in our test and this could explain the apparent normal levels of aac in the children with positive rheumatoid factor JCA, but the decomplementing step, which entails removal of C1q with an insolublised IgG immunoabsorbate, would also remove rheumatoid factor, and so would lessen this effect. It is also possible, though, that this step might remove rheumatoid factor containing complexes. The negative finding in JCA with amyloidosis is an interesting new observation. Our data suggest that if the aac detected by this technique play a part in the pathogenesis of this disease, it is largely in the systemic manifestations.

Relatively low levels of serum $\operatorname{IgA}$ were detected in only 3 patients, and each had received drugs, such as penicillamine, which may have caused secondary IgA deficiency, so none of these patients is certainly among the minority of JCA with primary IgA deficiency (Cassidy et al., 1973, 1977; Barkley et al., 1978). The defect of yeast opsonisation is associated with a number of diseases which are also associated with IgA deficiency, so we measured it in these patients. The frequency $(10 \%)$ of values below our diagnostic threshold $(2 \cdot 5)$ is not significantly greater than that of a healthy population $(5 \%-$ Soothill and Harvey, 1976). Only one had a level of C2 which suggested heterozygosity for C2 deficiency; this is consistent with the reported incidence of such levels in the population and lower than in a previous report in JCA (Glass et al., 1976). Our findings do not suggest that these deficiencies contribute importantly to the aetiology of JCA in our patients, 
and we could not investigate the relationship of the different possible immunodeficiencies to the aac findings. We can only assume that our patients are different from those studied by Glass et al. (1976), and the role of other immunodeficiencies requires further study.

We thank the National Fund for Research into Crippling Disease for their grant to R.J.L.

\section{References}

Amman, A. J., and Hong, R. (1971). Selective IgA deficiency: presentation of 30 cases and review of the literature. Medicine, 50, 223-236.

Ansell, B. M., and Wood, P. (1976). Prognosis in juvenile chronic polyarthritis. Clinics in Rheumatic Diseases, 2, $397-412$.

Barkley, D., Hohermuth, H., Howard, A., Webster, D., and Ansell, B. M. (1978). IgA deficiency in juvenile chronic polyarthritis. Journal of Rheumatology (in press).

Bianco, N. E., Panush, R. S., Stillman, J. S., and Schur, P. H. (1971). Immunological studies of juvenile rheumatoid arthritis. Arthritis and Rheumatism, 14, 685-696.

Cassidy, J. T., Petty, R. E., and Sullivan, D. B. (1973). Abnormalities in the distribution of serum immunoglobulins. Journal of Clinical Investigation, 52, 1931-1936.

Cassidy, J. T., Petty, R. E., and Sullivan, D. B. (1977). Occurrence of selective IgA deficiency in children with juvenile rheumatoid arthritis. Arthritis and Rheumatism, 20, Supplement 2, 181-183.

Fahey, J. L., and McKelvey, E. M. (1965). Quantitative determination of serum immunoglobulins in antibodyagar plates. Journal of Immunology, 94, 84-90.

Glass, D., Raum, D., Gibson, D., Stillman, J. S., and Schur, P. H. (1976). Inherited deficiency of the second component of complement. Journal of Clinical Investigation, 58, 853861.
Levinsky, R. J., and Soothill, J. F. (1977). A test for antigenantibody complexes in human sera using IgM of rabbit antisera to human immunoglobulins. Clinical and Experimental Immunology, 29, 428-435.

Miller, M. E., Seals, J., Kaye, R., and Levitsky, L. C. (1968). A familial plasma-associated defect of phagocytosis: a new cause of recurrent bacterial infections. Lancet, 2 , 60-63.

Munthe, E., and Natvig, J. B. (1972). Immunoglobulin classes, subclasses, and complexes of IgG rheumatoid factor in rheumatoid plasma cells. Clinical and Experimental Immunology, 12, 55-70.

Nydegger, U. E., Ackermann, L. M., Lambert, P. H., and Miescher, P. A. (1972). A simple automated method for complement estimation in a continuous flow system.Journal of Immunology, 109, 910-915.

Rossen, R. D., Brewer, E. J., Person, D. A., Templeton, J. W., and Lidsky, M. D. (1977). Circulating immune complexes and antinuclear antibodies in juvenile rheumatoid arthritis. Arthritis and Rheumatism, 20, 1485-1490.

Soothill, J. F. (1976). Some intrinsic and extrinsic factors predisposing to allergy. Proceedings of the Royal Society of Medicine, 69, 439-442.

Soothill, J. F., and Steward, M. W. (1971). The immunopathological significance of the heterogeneity of antibody affinity. Clinical and Experimental Immunology, 9, 193199.

Soothill, J. F., and Harvey, B. A. M. (1976). Defective opsonization. A common immunity deficiency. Archives of Disease in Childhood, 51, 91-99.

Soothill, J. F., and Harvey, B. A. M. (1977). A defect of the alternative pathway of complement. Clinical and Experimental Immunology, 27, 30-33.

Correspondence to Professor J. F. Soothill, Department of Immunology, Institute of Child Health, 30 Guilford Street, London WC1N 1EH.

Received 30 May 1978 